Yukawa Institute Kyoto

YITP-02-39

hep-th/0208005

October 2002

\title{
Sine-Gordon quantum field theory on the half-line with quantum boundary degrees of freedom
}

\author{
P. Baseilhac ${ }^{1}$ and K. Koizumi \\ ${ }^{1}$ Yukawa Institute for Theoretical Physics \\ Kyoto University, Kyoto 606-8502, Japan \\ ${ }^{2}$ Department of Physics, \\ Kyoto Sangyo University, Kyoto 603-8555, Japan
}

\begin{abstract}
The sine-Gordon model on the half-line with a dynamical boundary introduced by Delius and one of the authors is considered at quantum level. Classical boundary conditions associated with classical integrability are shown to be preserved at quantum level too. Non-local conserved charges are constructed explicitly in terms of the field and boundary operators. We solve the intertwining equation associated with a certain coideal subalgebra of $U_{q}\left(\widehat{s l_{2}}\right)$ generated by these non-local charges. The corresponding solution is shown to satisfy quantum boundary Yang-Baxter equations. Up to an exact relation between the quantization length of the boundary quantum mechanical system and the sine-Gordon coupling constant, we conjecture the soliton/antisoliton reflection matrix and bound states reflection matrices. The structure of the boundary state is then considered, and shown to be divided in two sectors. Also, depending on the sine-Gordon coupling constant a finite set of boundary bound states are identified. Taking the analytic continuation of the coupling, the corresponding boundary sinh-Gordon model is briefly discussed. In particular, the particle reflection factor enjoys weak-strong coupling duality.
\end{abstract}

PACS: 10.10.-z; 11.10.Kk; 11.25.Hf; 64.60.Fr

Keywords: Massive boundary integrable field theory; sine-Gordon model; boundary degrees of freedom; reflection equations, reflection matrix

\section{Introduction}

Two-dimensional quantum field theories with boundary have attracted attention for many years, as they play an important role in the analysis of low dimensional statistical systems near criticality or quantum gravity (open string approach). Solving integrable theories restricted on the half-line for various types of boundary is then of great interest. Among the known examples, the sine-Gordon model with a non-dynamical boundary has been shown to be integrable at classical level $[1,2]$ as well as at quantum level $[3,4]$. This has provided one of the simplest examples for which exact results such as factorized scattering theory, boundary spectrum, etc...[5, 6,7 , $8,9,10,11,12,13,14,15,16,17]$ have been derived.

\footnotetext{
${ }^{1}$ pascal@yukawa.kyoto-u.ac.jp

${ }^{2}$ kkoizumi@cc.kyoto-su.ac.jp
} 
Recently [18], an integrable Hamiltonian which describes a sine-Gordon model on the half-line coupled to a non-linear oscillator at the boundary has been shown to be integrable at classical level. Using a solution of the classical reflection equations (also called classical boundary Yang-Baxter equations) and following Sklyanin formalism we have obtained an infinite number of mutually commuting classical conserved quantities, provided some specific classical boundary conditions. The existence of such integrals of motion is a sufficient condition which ensures classical integrability of the model. Quantum integrability had remained an open question. Although a solution of the quantum reflection equations - quantum boundary Yang-Baxter equations (qBYBE) - had been proposed for a certain representation of the quantum $R$-matrix $[19,20]$, there was no reason to believe that this solution could describe the soliton/antisoliton scattering process bouncing off the boundary.

Independently, the method of construction of non-local conserved charges in integrable models in the bulk [21] has been extended to non-dynamical boundary ones more recently. For instance, the sine-Gordon model [22] and the $A_{r}^{(1)}$ affine Toda theory with imaginary coupling [23] restricted to the half-line have been studied. The existence of such non-local conserved charges provides a useful tool to determine the scattering properties of the theory. Indeed, the explicit form of the $S$-matrix (soliton/antisoliton scattering) and the $K$-matrix (soliton/antisoliton scattering on the boundary) is encoded in the minimal solution of quantum Yang-Baxter equations (qYBE)s and qBYBEs, respectively.

In this paper, we study at quantum level the model introduced in [18]. In section 2 we will show that quantum boundary conditions take a form similar to the classical boundary ones proposed in [18]. Also, we will construct non-local conserved charges corresponding to our dynamical case, which are natural extensions of the known ones (non-dynamical) $[22,23]$. In our case the non-local conserved charges generate a certain coideal subalgebra of $U_{q}\left(\widehat{s l_{2}}\right)$ mixed with the Heisenberg one, where $q$ is the deformation parameter. Further, by specifying the representation for $U_{q}\left(\widehat{s l_{2}}\right)$ we solve the corresponding intertwining equations. It provides a new solution $K_{0}(\theta)$ to the qBYBEs, different from the one proposed in $[19,20,18]$.

In section 4 , we use the minimal solution $K_{0}(\theta)$ of these equations ${ }^{3}$ to construct the soliton/antisoliton reflection matrix $K_{S G}^{(\epsilon)}(\theta)$ which describes the scattering of the sine-Gordon soliton/antisoliton off the dynamical boundary. In particular, taking the commutative limit of the Heisenberg algebra, we check that the "minimal" part reduces to the one obtained by Ghoshal-Zamolodchikov [3] and DeVega-Gonzales-Ruiz [24]. In the generic noncommutative case, we show that $K_{S G}^{(\epsilon)}(\theta)$ is a solution of the qBYBEs associated with the sine-Gordon $S$ matrix. This shows consistency of the nonperturbative analysis based on non-local conserved charges approach. To determine uniquely $K_{S G}^{(\epsilon)}(\theta)$ we impose the boundary unitarity condition as well as the boundary cross-unitarity condition proposed in [3]. Studying the singularities of the proposed reflection matrix we identify a finite set of boundary bound states. Then, we apply the bootstrap equation to construct explicitly the reflection matrices associated with bound states (breathers) scattering off the boundary.

As we are going to see, with the help of certain algebraic relations between Heisenberg operators the boundary unitarity condition is satisfied without specifying the boundary state structure. However, the boundary crossunitarity condition leads to restrict its form in order to preserve certain "physical" principles. We identify the corresponding constraints: choosing a continuous representation for the boundary operators difference equations are obtained. They restrict the boundary state in two sectors, associated with "even" or "odd" breathers $B_{n}$.

Concluding remarks follow in the last section. There, following Corrigan [12] we use the so-called breatherparticle identification to obtain the particle reflection factor in the boundary sinh-Gordon model with quantum degrees of freedom at the boundary. The result is found to be self-dual under the weak-strong coupling duality transformation $b \leftrightarrow 2 / b$.

\section{Classical sine-Gordon field theory with a dynamical boundary}

Let us first recapitulate some main results of [18] without details. There, we considered a Hamiltonian describing the interaction between a sine-Gordon field theory restricted to the half-line and a non-linear oscillator living at the boundary. The sine-Gordon part describes a relativistic $1+1$ dimensional self-interacting bosonic field $\phi(x, t)$

\footnotetext{
${ }^{3}$ These relations, in the dynamical case, are no longer linear in terms of the whole set of operators.
} 
with mass $m$ and its conjugate field $\pi(x, t)$. The Hamiltonian reads

$$
\begin{aligned}
H_{S G}=\int_{-\infty}^{0} d x & \left(\frac{1}{2} \pi(x)^{2}+\frac{1}{2}\left(\partial_{x} \phi(x)\right)^{2}-\frac{m^{2}}{\beta^{2}} \cos \beta \phi(x)\right) \\
& -\frac{2 m}{\beta^{2}}\left(\cos \left(\beta \tilde{p} / \sqrt{2 M_{0} m}\right) e^{-i \beta \phi(0) / 2}+\cos \left(\beta \sqrt{M_{0} m} \tilde{q} / 2 \sqrt{2}\right) e^{i \beta \phi(0) / 2}\right) .
\end{aligned}
$$

Expanding the oscillator part for small $\tilde{q}$ and $\tilde{p}$, these degrees of freedom can be interpreted as position and momentum variables, respectively. In this limit, it can be shown that the effective mass of the oscillator depends on the value of the field at the boundary and the parameter $M_{0}$.

At fixed time $t$ we introduced the Poisson bracket

$$
\left\{\mathcal{O}_{1}, \mathcal{O}_{2}\right\}=\int_{-\infty}^{0} d x\left[\frac{\delta \mathcal{O}_{1}}{\delta \pi(x)} \frac{\delta \mathcal{O}_{2}}{\delta \phi(x)}-\frac{\delta \mathcal{O}_{1}}{\delta \phi(x)} \frac{\delta \mathcal{O}_{2}}{\delta \pi(x)}\right]+\frac{\partial \mathcal{O}_{1}}{\partial \tilde{p}} \frac{\partial \mathcal{O}_{2}}{\partial \tilde{q}}-\frac{\partial \mathcal{O}_{1}}{\partial \tilde{q}} \frac{\partial \mathcal{O}_{2}}{\partial \tilde{p}}
$$

for any observable $\mathcal{O}_{j}$. At constant time slices the non-vanishing Poisson brackets in the sine-Gordon field theory and for the boundary variables $\tilde{p}, \tilde{q}$ are, respectively

$$
\{\pi(x), \phi(y)\}=\delta(x-y), \quad\{\tilde{p}, \tilde{q}\}=1
$$

from which it is straightforward to calculate the equations of motion. However, in order to have the continuity of these equations on the left half-line ] $-\infty, 0]$, the equations for the field $\pi(x)$ leads to the classical boundary condition

$$
\partial_{x} \phi(0)=-i \frac{m}{\beta}\left(\cos \hat{p} e^{-i \beta \phi(0) / 2}-\cos \hat{q} e^{i \beta \phi(0) / 2}\right) .
$$

where we have introduced the reduced parameters $\hat{p}=\frac{\beta}{\sqrt{2 M_{0} m}} \tilde{p}$ and $\hat{q}=\frac{\beta \sqrt{M_{0} m}}{2 \sqrt{2}} \tilde{q}$.

The model with Hamiltonian (1) is integrable: it is possible to construct an infinite number of higher spin integrals of motion that are in involution with each other. For instance, assuming the boundary condition above (4) the first non-trivial integral of motion reads

$$
\begin{aligned}
I_{3}= & \int_{-\infty}^{0}\left(\frac{\beta^{4}}{16 m^{3}}\left(\pi(x)^{4}+6 \pi(x)^{2}\left(\partial_{x} \phi(x)\right)^{2}+\left(\partial_{x} \phi(x)\right)^{4}\right)-\frac{\beta^{2}}{m^{3}}\left(\left(\partial_{x} \pi(x)\right)^{2}+\left(\partial_{x}^{2} \phi(x)\right)^{2}\right)\right. \\
& \left.-\frac{\beta^{2}}{4 m}\left(\pi(x)^{2}+5\left(\partial_{x} \phi(x)\right)^{2}\right) \cos \beta \phi(x)+\frac{m}{8}(\cos 2 \beta \phi(x))\right) d x+I_{3}^{\text {boundary }}
\end{aligned}
$$

with

$$
\begin{aligned}
I_{3}^{\text {boundary }} & =e^{3 i \beta \phi(0) / 2}\left(\frac{1}{2} \cos \hat{q}+\frac{1}{6} \cos ^{3} \hat{q}\right)-e^{i \beta \phi(0) / 2}\left(\frac{3}{2} \cos \hat{p}-\frac{1}{2} \cos ^{2} \hat{q} \cos \hat{p}+\frac{\beta^{2}}{2 m^{2}} \pi(0)^{2} \cos \hat{q}\right) \\
& +e^{-3 i \beta \phi(0) / 2}\left(\frac{1}{2} \cos \hat{p}+\frac{1}{6} \cos ^{3} \hat{p}\right)-e^{-i \beta \phi(0) / 2}\left(\frac{3}{2} \cos \hat{q}-\frac{1}{2} \cos ^{2} \hat{p} \cos \hat{q}+\frac{\beta^{2}}{2 m^{2}} \pi(0)^{2} \cos \hat{p}\right) \\
& -i \frac{2 \beta}{m} \sin \hat{q} \sin \hat{p} .
\end{aligned}
$$

In general, this Hamiltonian is not real. Such kind of situation is typical in higher rank affine Toda theories with imaginary coupling for which the Hamiltonian is also non-hermitian. In these theories, the classical soliton solutions are found to be complex. Nevertheless, the energy of these configurations is real [25].

\section{Quantum sine-Gordon field theory with a quantum boundary}

Let us suppose that there exists a well-defined action describing the sine-Gordon (SG) field theory restricted on the half-line coupled with a quantum mechanical system at the boundary. To characterize the dynamics of the boundary we introduce, let say, two boundary operators $\mathcal{E}_{ \pm}(y)$. We propose the following action

$$
\mathcal{A}=\int_{-\infty}^{\infty} d y \int_{-\infty}^{0} d x\left(\frac{1}{8 \pi}\left(\partial_{\nu} \phi\right)^{2}-2 \mu \cos (\hat{\beta} \phi)\right)+\mu^{1 / 2} \int_{-\infty}^{\infty} d y \Phi_{\text {pert }}^{B}(y)+\mathcal{A}_{\text {boundary }}
$$


where the interaction between the field and the dimensionless boundary quantum operators reads

$$
\Phi_{\text {pert }}^{B}(y)=\mathcal{E}_{-}(y) e^{i \hat{\beta} \phi(0, y) / 2}+\mathcal{E}_{+}(y) e^{-i \hat{\beta} \phi(0, y) / 2}
$$

and $\mathcal{A}_{\text {boundary }}$ is the kinetic part associated with the boundary operators. Here we used the notation $\hat{\beta}=\beta / \sqrt{4 \pi}$. In the deep UV, this model can be considered as a relevant perturbation of a conformal field theory (for instance the free Gaussian field) on the semi-infinite plane with certain boundary conditions at $x=0$. In the $\operatorname{limit}^{4} \mu=0$ Neumann boundary conditions $\left.\partial_{x} \phi\right|_{x=0}=0$ preserve integrability. If one turns on the boundary perturbation $(\mu \neq 0)$, following the arguments of [3] we expect that a quantum analogue of the classical integral of motion (5) can be constructed explicitly. Obviously, the boundary operators $\hat{\mathcal{E}}_{ \pm}(y)$ will have to satisfy certain algebraic relations in order to preserve integrability at quantum level too. Even if we do not construct explicitly such local quantum conserved charges here, analysis of following sections will support integrability of the model.

There are two alternative description ${ }^{5}$ of the model (6). On one hand, one can choose the $y$-direction to be the "time" in which case the Hamiltonian $H_{B}$ contains the boundary contribution, and the Hilbert space $\mathcal{H}_{B}$ is identified with the half-line $y=$ Const.. Then, correlation functions are calculated over the ground state of $H_{B}$, denoted $|0\rangle_{B}$ below.

On the other hand, one can choose the infinite line $x=$ Const. as the "equal time section". The Hamiltonian in this case is the same as in the bulk theory on the full line with Hilbert space $\mathcal{H}$. The boundary at $x=0$ plays the role of initial condition, and all its information is encoded in the boundary state $|B\rangle \in \mathcal{H}$. Correlation functions are written as $\langle 0|\ldots| B\rangle$ where $|0\rangle \in \mathcal{H}$ is a (degenerate) ground state of $\mathcal{H}$. Notice that the existence of local integrals of motion $\mathcal{I}^{(s)}, \overline{\mathcal{I}}^{(s)}$ for certain values of $s$ implies

$$
\left(\mathcal{I}^{(s)}-\overline{\mathcal{I}}^{(s)}\right)|B\rangle=0
$$

In the perturbed boundary conformal field theory (BCFT) approach, the free bosonic fundamental field restricted to the half-line can be written in terms of its holomorphic/anti-holomorphic components

$$
\phi(x, y)=\varphi(z)+\bar{\varphi}(\bar{z}) .
$$

If we denote the expectation value in the BCFT with Neumann boundary conditions $\langle\ldots\rangle_{0}$, then the holomorphic components are normalized such that

$$
\langle\varphi(z) \varphi(w)\rangle_{0}=-2 \ln (z-w), \quad\langle\bar{\varphi}(\bar{z}) \bar{\varphi}(\bar{w})\rangle_{0}=-2 \ln (\bar{z}-\bar{w}), \quad\langle\varphi(z) \bar{\varphi}(\bar{w})\rangle_{0}=-2 \ln (z-\bar{w}) .
$$

To find the boundary conditions at quantum level, we can consider the expectation value of the local field $\partial_{x} \phi(0, y)$ with any other local field in first order of boundary conformal perturbation theory $\mu \rightarrow 0$. Due to the presence of the boundary operators $\mathcal{E}_{ \pm}(y)$, we write the ground state $|0\rangle_{B}$ as

$$
|0\rangle_{B}=|v a c\rangle_{B} \otimes|0\rangle_{B C F T}+\mathcal{O}(\mu)
$$

where $|0\rangle_{B C F T} \in \mathcal{H}_{B C F T}$ and $|v a c\rangle_{B}$ denotes the effective boundary ground state which satisfies the Schrödinger equation

$$
H\left(\mathcal{E}_{+}, \mathcal{E}_{-}\right)|v a c\rangle_{B}=E_{0, B}^{e f f}|v a c\rangle_{B}
$$

with effective ground state ${ }^{6}$ energy $E_{0, B}^{e f f}$. Here, $H\left(\mathcal{E}_{+}, \mathcal{E}_{-}\right)$is an effective boundary Hamiltonian. In this approximation, following the analysis of [23] with eqs. (8) it is straightforward to show that, in first order of boundary conformal perturbation theory, the quantum boundary condition takes a form similar to the classical

\footnotetext{
${ }^{4}$ As we will see later, integrability of the QFT (6) requires that one can not turn on the boundary perturbation independently of $\mu$.

${ }^{5}$ It seems important to us to recall the analysis of [3].

${ }^{6}$ Taking the perturbative limit $\hat{\beta} \rightarrow 0$ and using an explicit realization of the boundary operators in terms of Heisenberg operators (see further sections), one can show that the "pure" boundary wave function satisfies certain difference equations.
} 
one. Furthermore, using scaling arguments ${ }^{7}$ it is possible to show that this condition is satisfied at all order in perturbation theory. It reads

$$
{ }_{B}\left\langle 0\left|\partial_{x} \phi(0, y) \ldots\right| 0\right\rangle_{B}=i 2 \pi \hat{\beta} \mu^{1 / 2}{ }_{B}\left\langle 0\left|\left(\mathcal{E}_{-}(y) e^{i \hat{\beta} \phi(0, y) / 2}-\mathcal{E}_{+}(y) e^{-i \hat{\beta} \phi(0, y) / 2}\right) \ldots\right| 0\right\rangle_{B} .
$$

The method of constructing non-local conserved charges in the model (6) follows the line presented in [23]. So, we refer the reader to this paper for more details. Here, the main difference is that $\mathcal{E}_{ \pm}(y)$ are operators. If we denote the "bulk" non-local charges [21]

$$
Q_{ \pm}=\frac{1}{4 \pi} \int_{-\infty}^{\infty} d x\left(J_{ \pm}-H_{ \pm}\right), \quad \bar{Q}_{ \pm}=\frac{1}{4 \pi} \int_{-\infty}^{\infty} d x\left(\bar{J}_{ \pm}-\bar{H}_{ \pm}\right),
$$

they can be expressed in terms of holomorphic/anti-holomorphic part of vertex operators as ${ }^{8}$

$$
\begin{array}{ll}
J_{ \pm}=: \exp \left( \pm \frac{2 i}{\hat{\beta}} \tilde{\varphi}\right): & \text { and } \quad H_{ \pm}=-4 \pi \mu \frac{\hat{\beta}^{2}}{\hat{\beta}^{2}-2}: \exp \left( \pm i\left(\frac{2}{\hat{\beta}}-\hat{\beta}\right) \tilde{\varphi} \mp i \hat{\beta} \tilde{\bar{\varphi}}\right): \\
\bar{J}_{ \pm}=: \exp \left(\mp \frac{2 i}{\hat{\beta}} \tilde{\bar{\varphi}}\right): & \text { and } \quad \bar{H}_{ \pm}=-4 \pi \mu \frac{\hat{\beta}^{2}}{\hat{\beta}^{2}-2}: \exp \left(\mp i\left(\frac{2}{\hat{\beta}}-\hat{\beta}\right) \tilde{\bar{\varphi}} \mp i \hat{\beta} \tilde{\varphi}\right): .
\end{array}
$$

Together with the "bulk" topological charge $T_{b u l k}=\frac{\hat{\beta}}{2 \pi} \int_{-\infty}^{\infty} d x \partial_{x} \tilde{\phi}$ they generate the quantum enveloping algebra $U_{q}\left(\widehat{s l_{2}}\right)$. In the theory on the half-line, these charges are no longer conserved. Instead, using (8) and the method of $[22,23]$ it is straightforward to show that the two combinations

$$
\hat{Q}_{ \pm}=Q_{ \pm}+\bar{Q}_{\mp}+\hat{\mathcal{E}}_{ \pm}(y) q^{\mp T} \quad \text { with } \quad T=\frac{\hat{\beta}}{2 \pi} \int_{-\infty}^{0} d x \partial_{x} \phi
$$

and

$$
\hat{\mathcal{E}}_{ \pm}(y)=\mu^{1 / 2} \frac{\hat{\beta}^{2}}{1-\hat{\beta}^{2}} \mathcal{E}_{ \pm}(y)
$$

are conserved to all orders in boundary conformal perturbation theory framework. Here, the deformation parameter is defined by

$$
q \equiv \exp \left(-2 i \pi / \hat{\beta}^{2}\right)
$$

As the sine-Gordon model possesses a single two dimensional soliton multiplet denoted by $\left|\psi_{ \pm}(\theta)\right\rangle$, we are now interested in two dimensional representations of $U_{q}\left(\widehat{s l_{2}}\right)$ on asymptotic soliton states, denoted $\pi_{\theta}$ below. Here, $\theta$ denotes the rapidity of the soliton/antisoliton. To determine the meaner in which non-local conserved charges (11) are represented on asymptotic single-soliton states, we use the notation of [21]. Furthermore, in the asymptotic limit $y \rightarrow \pm \infty$ on the boundary we will assume

$$
\hat{\mathcal{E}}_{+}(y= \pm \infty) \equiv \hat{\mathcal{E}}_{+} \quad \text { and } \quad \hat{\mathcal{E}}_{-}(y= \pm \infty) \equiv \hat{\mathcal{E}}_{-} .
$$

In the first Hamiltonian picture $y$ is identified with the time axis. In other words, the condition (14) means that boundary operators lead to the same operators in the far past and future. We must keep in mind that $\hat{\mathcal{E}}_{ \pm}$are operators satisfying $\left[\hat{\mathcal{E}}_{ \pm}, g\right]=0$ and $\pi_{\theta}\left(\hat{\mathcal{E}}_{ \pm} g\right)=\hat{\mathcal{E}}_{ \pm} \pi_{\theta}(g), \forall g \in U_{q}\left(\widehat{s l_{2}}\right)$. Thus we have

$$
\pi_{\theta}\left(\hat{Q}_{ \pm}\right)_{+}^{+}=\hat{\mathcal{E}}_{ \pm} q^{\mp 1}, \quad \pi_{\theta}\left(\hat{Q}_{ \pm}\right)_{-}^{+}=c e^{ \pm \lambda \theta}, \quad \pi_{\theta}\left(\hat{Q}_{ \pm}\right)_{+}^{-}=c e^{\mp \lambda \theta}, \quad \pi_{\theta}\left(\hat{Q}_{ \pm}\right)_{-}^{-}=\hat{\mathcal{E}}_{ \pm} q^{ \pm 1}
$$

with

$$
\lambda=2 / \hat{\beta}^{2}-1 \quad \text { and } \quad c^{2}=i 2 \mu\left(q^{2}-1\right) / \lambda^{2} .
$$

\footnotetext{
${ }^{7}$ Dimensions of both side must be equal. Due to the form of the perturbing operator one finds that the only term that can appear on the right hand side must be linear in the parameter $\mu$. All other terms must vanishes.

${ }^{8}$ On the contrary to [23], here we denote the fundamental field one the whole line $\tilde{\phi}(x, y)$ and the one restricted to the half-line $\phi(x, y)$. Then, the chiral components are related in the following way: $\varphi(x, y)=\tilde{\varphi}(x, y)+\tilde{\bar{\varphi}}(-x, y), \quad \bar{\varphi}(x, y)=\tilde{\bar{\varphi}}(x, y)+\tilde{\varphi}(-x, y)$.
} 
For the remaining symmetry algebra of the QFT (6) generated by the non-local conserved charges given above (11) we are looking for a solution to the intertwining equation

$$
K_{0}^{\delta}(\theta) \pi_{\theta}\left(\hat{Q}_{ \pm}\right)_{\zeta}^{\nu}=\pi_{-\theta}\left(\hat{Q}_{ \pm}\right)_{\nu}^{\delta} K_{0 \zeta}^{\nu}(\theta)
$$

where indices $\{\delta, \nu, \zeta\} \in\{ \pm\}$ refer to two dimensional representations of $U_{q}\left(\widehat{s l_{2}}\right)$ on asymptotic soliton states $\left|\psi_{ \pm}(\theta)\right\rangle$. Then, using this representation the solution $K_{0}(\theta)$ is written as a $2 \times 2$ matrix with entries expressed in terms of the boundary operators. In the non-dynamical case, the entries are just analytic functions of $\theta$ as $\mathcal{E}_{ \pm}$ are $c$-numbers. For further convenience, let us define

$$
\begin{aligned}
K_{0_{+}^{+}}^{+}(\theta) & =A(\theta), & & K_{0_{-}^{+}}^{+}(\theta)=B(\theta), \\
K_{0+}^{-}(\theta) & =D(\theta), & & K_{0_{-}^{-}}^{-}(\theta)=E(\theta) .
\end{aligned}
$$

After some calculations, we find that the entries of the minimal solution $K_{0}(\theta)$ of the intertwining property (16) takes the following form:

$$
\begin{aligned}
& A(\theta)=\left(q^{-1} e^{\lambda \theta} \hat{\mathcal{E}}_{+}-q e^{-\lambda \theta} \hat{\mathcal{E}}_{-}\right)\left(q-q^{-1}\right) / 2 c \\
& E(\theta)=\left(q^{-1} e^{\lambda \theta} \hat{\mathcal{E}}_{-}-q e^{-\lambda \theta} \hat{\mathcal{E}}_{+}\right)\left(q-q^{-1}\right) / 2 c \\
& B(\theta)=\left(-c^{2} q^{-1} e^{2 \lambda \theta}-c^{2} q e^{-2 \lambda \theta}+\frac{q-q^{-1}}{q+q^{-1}}\left(q^{-1} \hat{\mathcal{E}}_{-} \hat{\mathcal{E}}_{+}-q \hat{\mathcal{E}}_{+} \hat{\mathcal{E}}_{-}\right)\right) / 2 c^{2}, \\
& D(\theta)=\left(-c^{2} q^{-1} e^{2 \lambda \theta}-c^{2} q e^{-2 \lambda \theta}+\frac{q-q^{-1}}{q+q^{-1}}\left(-q \hat{\mathcal{E}}_{-} \hat{\mathcal{E}}_{+}+q^{-1} \hat{\mathcal{E}}_{+} \hat{\mathcal{E}}_{-}\right)\right) / 2 c^{2}
\end{aligned}
$$

if the boundary operators satisfy certain commutation relations with the entries $B(\theta)$ and $D(\theta)$. Among these, we obtain the commutation relation ${ }^{9}$

$$
\left[\hat{\mathcal{E}}_{+} \hat{\mathcal{E}}_{-}, \hat{\mathcal{E}}_{-} \hat{\mathcal{E}}_{+}\right]=c^{2}\left(q+q^{-1}\right)^{2}\left(\hat{\mathcal{E}}_{+}^{2}-\hat{\mathcal{E}}_{-}^{2}\right) .
$$

Here, we propose a realization of the boundary operators at $y= \pm \infty$ in terms of operators $\{\hat{p}, \hat{q}\}$ which belong to the Heisenberg algebra $[\hat{p}, \hat{q}] \sim \alpha$ in order to have (19) satisfied. Due to (14) we denote $\hat{p}( \pm \infty) \equiv \hat{p}$ and $\hat{q}( \pm \infty) \equiv \hat{q}$. Indeed, one can check that setting ${ }^{10}$

$$
\hat{\mathcal{E}}_{+}= \pm 2 e_{U V} \cosh \hat{p} \quad \text { and } \quad \hat{\mathcal{E}}_{-}= \pm 2 e_{U V} \cosh \hat{q} \quad \text { where } \quad \pm q=\exp (\alpha / 2)
$$

with the normalization $e_{U V}^{2}=-c^{2} /\left(q-q^{-1}\right)^{2}$, all the equations (16) can be satisfied for a certain relation between the SG coupling constant and the boundary quantization length $\alpha$. However, according to eqs. (20) there is an ambiguity in the definition of the sign of the boundary perturbation. Depending on each sign, the boundary quantization length is fixed to

$$
[\hat{p}, \hat{q}]=\alpha \bmod (4 i \pi) \quad \text { with } \quad \alpha=i 4 \pi \frac{\left(\hat{\beta}^{2}-1\right)}{\hat{\beta}^{2}} \quad(+) \quad \text { or } \quad \alpha=i 2 \pi \frac{\left(\hat{\beta}^{2}-2\right)}{\hat{\beta}^{2}} \quad(-) .
$$

In the model (6) with (12) and (20), $e_{U V}$ is fixed by the "bulk" non-local conserved charges algebraic structure which gives

$$
e_{U V}^{2}=\frac{i 2\left(1-q^{2}\right)}{\lambda^{2}\left(q-q^{-1}\right)^{2}} \mu
$$

To relate the UV parameter $e_{U V}$ and the IR soliton mass parameter $M$ we can use their exact relation calculated in $[26]$ :

$$
\mu=\frac{\Gamma\left(\hat{\beta}^{2} / 2\right)}{\pi \Gamma\left(\left(2-\hat{\beta}^{2}\right) / 2\right)}\left[\frac{M \sqrt{\pi} \Gamma(1 / 2+1 / 2 \lambda)}{2 \Gamma(1 / 2 \lambda)}\right]^{2-\hat{\beta}^{2}} .
$$

${ }^{9}$ For generic values of the coupling and explicit boundary operators dependence in the boundary scattering description, this condition is necessary. Also, we do not assume specific values of the coupling here (e.g. free fermion point, reflectionless point), for which new solutions may be constructed.

${ }^{10}$ Notice that this solution also works, obviously, if one changes $\alpha \rightarrow-\alpha$. 
It follows that the strength of the boundary perturbation is fixed by the bulk soliton mass $M$. This is consistent with the classical model (1) in the sense that the only free parameter is the mass $m^{11}$. In other words, choosing an arbitrary boundary mass would destroy integrability. Physically, this phenomena is not so surprising: to preserve integrability the effects brought by the SG model and the non-linear oscillator (energy transfer, for instance) have to compensate each other, which is characterized by the exact relation between the quantization length $\alpha$ and the SG coupling constant in (21).

At specific values of the SG coupling constant $\hat{\beta}^{2}=2 /(n+1)$ with $n \in \mathbb{N}$ the operators $\hat{\mathcal{E}}_{ \pm}$commute, and we have $q^{2}=1$. Then, there is no need to assume some special relation involving $c$ and the boundary parameters like $(19)$, and $\hat{\mathcal{E}}_{ \pm}$remain free. Consequently, $K_{0}(\theta)$ is reduced to a $2 \times 2$ matrix where $\hat{\mathcal{E}}_{ \pm}$are $c$-numbers. Expanding $K_{0}(\theta)$ in powers of $\left(q^{2}-1\right)$ we obtain

$$
K_{0}(\theta) \sim 2 \cosh (2 \lambda \theta) \mathbb{P} /\left(q-q^{-1}\right)+i K_{\text {non-dyn }}^{\min }(\theta),
$$

where $\mathbb{P}$ is the $2 \times 2$ permutation matrix. The first (singular) term is a trivial solution of the intertwining property (16) whereas the second term is the minimal reflection matrix obtained by Ghoshal-Zamolodchikov and DeVega-Gonzales-Ruiz [3, 24].

\section{Boundary Yang-Baxter equations and factorized scattering theory}

For the coupling constant $\hat{\beta}^{2}<2$, the bulk sine-Gordon model in $1+1$ Minkowski space-time is massive and integrable. The particle spectrum consists of a soliton/antisoliton pair $\left(\psi_{+}(\theta), \psi_{-}(\theta)\right)$ with mass $M$ and neutral particles, called "breathers", $B_{n}(\theta) \quad n=1,2, \ldots,<\lambda$. As usual, $E=M \cosh \theta$ and $P=M \sinh \theta$ the energy and momentum, respectively, of the soliton/antisoliton. In this model, $\mathcal{H}$ is the Fock space of multiparticle states. A general $N$-particles state is generated by the "particle creation operators" $A_{a_{i}}\left(\theta_{i}\right)$

$$
\left|A_{a_{1}}\left(\theta_{1}\right) \ldots A_{a_{N}}\left(\theta_{N}\right)\right\rangle=A_{a_{1}}\left(\theta_{1}\right) \ldots A_{a_{N}}\left(\theta_{N}\right)|0\rangle
$$

where $a_{i}$ characterizes the type of particle. The commutation relations between these operators are determined by the $S$-matrix elements, which describe the factorized scattering theory [27]. Integrability imposes strong constraints on the system which implies that the $S$-matrix has to satisfy the quantum Yang-Baxter equations. For instance, the SG soliton/antisoliton scattering $S$-matrix can be written as [27]

$$
S_{S G}(\theta)=R(\theta) \rho(-i \theta) / i
$$

where we introduced the four dimensional representation of the trigonometric solution of the quantum YangBaxter equation written in terms of the standard Pauli matrices $\sigma_{k}, k=1,2,3$ :

$$
R(\theta)=\frac{a(\theta)}{2}\left(I \otimes I+\sigma_{3} \otimes \sigma_{3}\right)+\frac{b(\theta)}{2}\left(I \otimes I-\sigma_{3} \otimes \sigma_{3}\right)+\frac{c(\theta)}{2}\left(\sigma_{1} \otimes \sigma_{1}+\sigma_{2} \otimes \sigma_{2}\right)
$$

with

$$
a(\theta)=\sinh (i \lambda \pi-\lambda \theta) \quad, \quad b(\theta)=\sinh (\lambda \theta) \quad, \quad c(\theta)=\sinh (i \lambda \pi) .
$$

The amplitudes $b(\theta)$ and $c(\theta)$ possess simple poles in the physical strip $0<\theta<i \pi$ located at $\theta_{n}=i \pi-i n \pi / \lambda$ for $n=1,2, \ldots<\lambda$. They are interpreted as the neutral bound state (breather) $B_{n}$. The factor $\rho(u)$ in $(25)$ ensures unitarity and crossing symmetry of the $S$-matrix. It reads

$$
\rho(u)=-\frac{1}{\pi} \Gamma(\lambda) \Gamma(1-\lambda u / \pi) \Gamma(1-\lambda+\lambda u / \pi) \prod_{l=1}^{\infty} \frac{F_{l}(u) F_{l}(\pi-u)}{F_{l}(0) F_{l}(\pi)},
$$

\footnotetext{
${ }^{11}$ Whatever the mass of the effective oscillator is, the relevant quantity is its frequency $\omega=m / 2$ [18]. Using the particle-breather identification, one can relate $m$ associated with the lightest breather with the mass $m$ of the fundamental particle.
} 
with

$$
F_{l}(u)=\frac{\Gamma(2 l \lambda-\lambda u / \pi) \Gamma(1+2 l \lambda-\lambda u / \pi)}{\Gamma((2 l+1) \lambda-\lambda u / \pi) \Gamma(1+(2 l-1) \lambda-\lambda u / \pi)} .
$$

Using the bootstrap equation, the amplitudes associated with (anti)soliton-breather $\psi_{ \pm} B_{n}$ and breather-breather $B_{n} B_{m}$ scattering have been calculated in [27].

\subsection{Soliton reflection matrix for the dynamical boundary}

Taking the first Hamiltonian picture, we can consider $y=i t$ in the QFT (6). If one uses the approach of [3], asymptotic states are now generated using $A_{a}(\theta)$ acting on the ground state $|0\rangle_{B} \in \mathcal{H}_{B}$. For boundary integrable field theories, the action of the creation operator on the boundary is characterized by the reflection matrix. It describes the scattering of the soliton/antisoliton on the boundary and is constrained by the so-called reflection equations, i.e. qBYBEs for a certain choice of representation. Depending of the quantization condition in (21) which determines the sign $\epsilon \in\{ \pm\}$ of the boundary operators (20) - the sine-Gordon reflection matrix $K_{S G}^{(\epsilon)}(\theta)$ must satisfy the qBYBEs

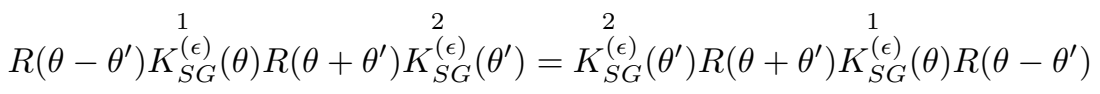

where we used the notations $\stackrel{1}{K}=K \otimes I, \stackrel{2}{K}=I \otimes K$. Recalling the definition of the entries (17) we have fourteen functional equations:

$$
\begin{aligned}
(i) & a_{-} c_{+}\left(B D^{\prime}-B^{\prime} D\right)+a_{-} a_{+}\left[A, A^{\prime}\right]=0, \\
(i i) & b_{-} b_{+}\left[A, E^{\prime}\right]+c_{-} c_{+}\left[E, E^{\prime}\right]+c_{-} a_{+}\left(D B^{\prime}-D^{\prime} B\right)=0, \\
(i i i) & c_{-} b_{+}\left(E A^{\prime}-E^{\prime} A\right)+b_{-} c_{+}\left(A A^{\prime}-E^{\prime} E\right)+b_{-} a_{+}\left[B, D^{\prime}\right]=0, \\
(i v) & b_{-} b_{+} A D^{\prime}+c_{-} c_{+} E D^{\prime}+c_{-} a_{+} D A^{\prime}-a_{-} a_{+} D^{\prime} A-a_{-} c_{+} E^{\prime} D=0, \\
(v) & b_{-} b_{+} B^{\prime} A+c_{-} c_{+} B^{\prime} E+c_{-} a_{+} A^{\prime} B-a_{-} a_{+} A B^{\prime}-a_{-} c_{+} B E^{\prime}=0, \\
(v i) & b_{-} b_{+} D^{\prime} E+c_{-} c_{+} D^{\prime} A+c_{-} a_{+} E^{\prime} D-a_{-} a_{+} E D^{\prime}-a_{-} c_{+} D A^{\prime}=0, \\
(v i i) & b_{-} b_{+} E B^{\prime}+c_{-} c_{+} A B^{\prime}+c_{-} a_{+} B E^{\prime}-a_{-} a_{+} B^{\prime} E-a_{-} c_{+} A^{\prime} B=0, \\
(v i i i) & b_{-} a_{+} B E^{\prime}+c_{-} b_{+} E B^{\prime}+b_{-} c_{+} A B^{\prime}-a_{-} b_{+} E^{\prime} B=0, \\
(i x) & b_{-} a_{+} A^{\prime} B+c_{-} b_{+} B^{\prime} A+b_{-} c_{+} B^{\prime} E-a_{-} b_{+} B A^{\prime}=0, \\
(x) & b_{-} a_{+} E^{\prime} D+c_{-} b_{+} D^{\prime} E+b_{-} c_{+} D^{\prime} A-a_{-} b_{+} D E^{\prime}=0, \\
(x i) & b_{-} a_{+} D A^{\prime}+c_{-} b_{+} A D^{\prime}+b_{-} c_{+} E D^{\prime}-a_{-} b_{+} A^{\prime} D=0,
\end{aligned}
$$

where we used the shorthand notations $a_{-}=a\left(\theta-\theta^{\prime}\right), a_{+}=a\left(\theta+\theta^{\prime}\right)$ and similarly for $b$ and $c$ as well as $A=A(\theta)$ and $A^{\prime}=A\left(\theta^{\prime}\right)$ and similarly for $B, D$ and $E$. The remaining three equations are obtained from $(i),(i i),($ iii $)$ through the substitutions $A \leftrightarrow E$ and $B \leftrightarrow D$. Straightforward calculations show that $K_{S G}^{(\epsilon)}(\theta)$ takes a form similar to $K_{0}(\theta)$. Indeed, the reflection matrix in the dynamical boundary case can be written

$$
K_{S G}^{(\epsilon)}(\theta)=K_{0}^{(\epsilon)}(\theta) Y(\theta)
$$

where the function $Y(\theta)$ has to be determined using some physical assumptions (see below). In the two dimensional representation of $U_{q}\left(\widehat{s l_{2}}\right)$, the matrix $K_{0}^{(\epsilon)}$ is obtained from (18) with the following substitutions ${ }^{12}$ :

$$
\hat{\mathcal{E}}_{+} \rightarrow \epsilon \cosh \hat{p}, \quad \hat{\mathcal{E}}_{-} \rightarrow \epsilon \cosh \hat{q}, \quad q \rightarrow e^{i \pi \lambda} \quad \text { and } \quad c \rightarrow \sin (\pi \lambda) .
$$

Here, the quantization condition is given in (21). In [3], Ghoshal and Zamolodchikov proposed the use of the "boundary unitarity" and "boundary cross-unitarity" conditions to determine the overall factor $Y(\theta)$ associated

\footnotetext{
${ }^{12}$ Notice that for specific values of the SG coupling constant such that $q$ is a root of unity, it is easy to construct a finite dimensional representation for the operators $A, B, D, E$ in the reflection matrix.
} 
with a non-dynamical boundary. Then, let us first consider the boundary unitarity condition. Independently of any representation for the boundary ground state $|0\rangle_{B}$, in our case it is natural to consider the combination of operators

$$
K_{S G_{c}}^{(\epsilon)}(\theta) K_{S G b}^{(\epsilon)}{ }^{c}(-\theta)=\delta_{b}^{a} \mathbb{I} \quad \text { with } \quad\{a, b, c\} \in\{ \pm\}
$$

as a generalization to the dynamical boundary case of the condition associated with the non-dynamical one. Here, the operator $\mathbb{I}$ denotes the identity which acts trivially on the boundary ground state. In terms of the entries defined in (17) these relations becomes

$$
\begin{aligned}
& A(\theta) A(-\theta)+B(\theta) D(-\theta)=\mathbb{I} / Y(\theta) Y(-\theta), \\
& D(\theta) B(-\theta)+E(\theta) E(-\theta)=\mathbb{I} / Y(\theta) Y(-\theta), \\
& A(\theta) B(-\theta)+B(\theta) E(-\theta)=0, \\
& D(\theta) A(-\theta)+E(\theta) D(-\theta)=0 .
\end{aligned}
$$

As the entries are noncommuting between each others, in general one would expect the product (31) to be illdefined. However, due to the specific form of the boundary operators one can show that changing $\theta \rightarrow-\theta$ does not affect the relations above. Also, the first two relations give the same result. Then, using algebraic relations like

$$
\begin{aligned}
& \left(q^{2}+q^{-2}\right) \hat{\mathcal{E}}_{+} \hat{\mathcal{E}}_{-} \hat{\mathcal{E}}_{+}-\hat{\mathcal{E}}_{+}^{2} \hat{\mathcal{E}}_{-}-\hat{\mathcal{E}}_{-} \hat{\mathcal{E}}_{+}^{2}=-c^{2}\left(q+q^{-1}\right)^{2} \hat{\mathcal{E}}_{-} \\
& \left(q^{2}+q^{-2}\right) \hat{\mathcal{E}}_{-} \hat{\mathcal{E}}_{+} \hat{\mathcal{E}}_{-}-\hat{\mathcal{E}}_{+} \hat{\mathcal{E}}_{-}^{2}-\hat{\mathcal{E}}_{-}^{2} \hat{\mathcal{E}}_{+}=-c^{2}\left(q+q^{-1}\right)^{2} \hat{\mathcal{E}}_{+}
\end{aligned}
$$

after some calculations we arrive at the condition

$$
Y(\theta) Y(-\theta)=\left[\sinh ^{2}(2 \lambda \theta)-\sinh ^{2}(i \pi \lambda)\right]^{-1} .
$$

Compared to the non-dynamical boundary sine-Gordon, the situation here is rather simple: there are no free parameters left in the unitarity condition (32) appart from the coupling constant $\hat{\beta}^{2}$ which appears through $\lambda$.

Let us now consider the boundary cross-unitarity condition. In the alternative Hamiltonian description, $x$ is now interpreted as "time" and the space of states is the same as in the bulk theory, i.e. $\mathcal{H}$. The "initial" (boundary) condition at $x=0$ is encoded in $|B\rangle$. If the theory is integrable (7) must be satisfied. It follows that $|B\rangle$ is a superposition of asymptotic states of the bulk theory constituted by pairs of particles of equal mass but opposite rapidities [3]. Thus, we define the boundary state as

$$
|B\rangle=\mathcal{N}\left(\left|\mathrm{B}_{0}\right\rangle+\sum_{\{n\}} g^{n} B_{n}(0)\left|\mathrm{B}_{n}\right\rangle+\frac{1}{2} \int_{-\infty}^{+\infty} d \theta K^{(\epsilon)} \frac{b}{a}\left(i \frac{\pi}{2}-\theta\right) A_{a}(\theta) A_{b}(-\theta)\left|\mathrm{B}_{a b}\right\rangle+\ldots\right) .
$$

Here $\mathcal{N}$ is a normalization coefficient and the states $\left|\mathrm{B}_{a_{1} \ldots a_{N}}\right\rangle \in \mathcal{V}_{\mathrm{B}}$ where $\mathcal{V}_{\mathrm{B}}$ is a representation for boundary operators. The second term corresponds to the contribution from the zero-momentum particles, namely the breathers. The coefficients $g^{n}$ indicate their contribution. Considering in particular the soliton scattering, arguments of [3] can be applied and we assume the boundary cross-unitarity condition

$$
K_{S G \bar{a}}^{(\epsilon)}(i \pi / 2-\theta)=S_{S G}^{a b} a^{\prime} b^{\prime}(2 \theta) K_{S G \bar{b}^{\prime}}^{(\epsilon)}(i \pi / 2+\theta),
$$

as the "in" and "out" states are related through the $S$-matrix. Notice that this relation is linear in the boundary operators. For simplicity, let us introduce two meromorphic functions $Y_{0}(\theta)$ and $Y_{1}(\theta)$ which solve the functional equations $(u \equiv-i \theta)$ :

$$
\begin{aligned}
& Y_{0}(\theta) Y_{0}(-\theta)=1 \\
& Y_{1}(\theta) Y_{1}(-\theta)=\left[\sinh ^{2}(2 \lambda \theta)-\sinh ^{2}(i \pi \lambda)\right]^{-1} \quad \text { and } \quad Y_{1}(i \pi / 2-\theta)=Y_{1}(i \pi / 2+\theta) \text {. }
\end{aligned}
$$


Then, it is not difficult to show that for the choice

$$
Y(\theta)=Y_{0}(\theta) Y_{1}(\theta)
$$

in (29), the boundary unitarity and boundary cross-unitarity conditions are satisfied. Following Ghoshal-Zamolodchikov notations we finally obtain

$$
Y_{0}(\theta)=R_{0}(u) G_{0}(u)
$$

where we used [3]

$$
R_{0}(u)=\prod_{k=1}^{\infty}\left[\frac{\Gamma(4 \lambda k-2 \lambda u / \pi) \Gamma(1+4 \lambda(k-1)-2 \lambda u / \pi)}{\Gamma(\lambda(4 k-3)-2 \lambda u / \pi) \Gamma(1+\lambda(4 k-1)-2 \lambda u / \pi)} /(u \rightarrow-u)\right]
$$

and we introduced

$$
G_{0}(u)=\prod_{k=1}^{\infty}\left[\frac{\Gamma(1+(4 k-2) \lambda-2 \lambda u / \pi) \Gamma((4 k-2) \lambda-2 \lambda u / \pi)}{\Gamma((4 k-4) \lambda-2 \lambda u / \pi) \Gamma(1+4 k \lambda-2 \lambda u / \pi)} /(u \rightarrow-u)\right] .
$$

Notice that the factor $R_{0}(u)$ contains poles in the "physical strip" $0<u<\pi / 2$ located at $u_{n}=n \pi / 2 \lambda$ for $n=1, \ldots<\lambda$. They are associated with zero-momentum soliton-antisoliton bound states (see last section). Also, we find

$$
Y_{1}(\theta)=\frac{\sigma(\pi / 2+\pi \lambda / 2, u) \sigma(\pi \lambda / 2, u)}{\sin (\pi \lambda)}
$$

where [3]

$$
\sigma(x, v)=\frac{\cos x}{\cos (x+\lambda v)} \prod_{l=1}^{\infty}\left[\frac{\Gamma(1 / 2+(2 l-1) \lambda+x / \pi-\lambda v / \pi) \Gamma(1 / 2+(2 l-1) \lambda-x / \pi-\lambda v / \pi)}{\Gamma(1 / 2+(2 l-2) \lambda-x / \pi-\lambda v / \pi) \Gamma(1 / 2+2 l \lambda+x / \pi-\lambda v / \pi)} /(v \rightarrow-v)\right]
$$

satisfies the relations

$$
\sigma(x, v) \sigma(x,-v)=\cos ^{2} x /(\cos (x+\lambda v) \cos (x-\lambda v)), \quad \sigma(x, \pi / 2-v)=\sigma(x, \pi / 2+v) .
$$

From (29) it is clear that there are no resonance states in comparison with the non-dynamical SG model. However, the factors $\sigma(\pi / 2+\pi \lambda / 2, u)$ and $\sigma(\pi \lambda / 2, u)$ brings an infinite number of singularities at real values of $u$. In the "physical strip" $0<u<\pi / 2$, depending on the value of the SG coupling constant, they are located at:

$$
u_{k}^{\text {even }}=\frac{\pi}{2}-\frac{k \pi}{\lambda} \quad \text { for } \quad 0<k<\frac{\lambda}{2} \quad \text { and } \quad u_{k}^{\text {odd }}=\frac{\pi}{2}-\frac{(2 k-1) \pi}{2 \lambda} \quad \text { for } \quad 0<k<\frac{\lambda+1}{2} .
$$

Denoting $E_{0}$ as the ground state energy, we identify these poles as boundary bound states with energy $E_{k}^{\text {even }}-E_{0}=$

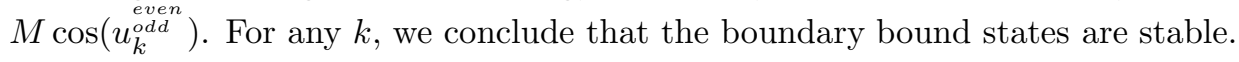

\subsection{Bound state reflection matrix for the dynamical boundary}

Above, we considered the reflection matrix of the soliton/antisoliton off the boundary. We now turn to the reflection matrix of the bound states (breathers) $B_{n}(\theta)$. To calculate it, we use the boundary bootstrap method introduced in $[3,5]$ and mainly follow [4]. Using "particle creation" operator formalism, the reflection matrix defines the commutation relation

$$
B_{n}(\theta) B=R_{B}^{(n)} B_{n}(-\theta) B
$$

where $B_{n}(\theta)$ creates the bound state $B_{n}$ with rapidity $\theta$. Then, the boundary bootstrap equation reads [3]

$$
f_{i_{1} i_{2}}^{n} K_{S G j_{1}}^{(\epsilon)}\left(\theta+\theta_{n} / 2\right) S_{S G_{j_{2} f_{1}}}^{i_{2} j_{1}}(2 \theta) K_{S G f_{2}}^{(\epsilon)}\left(\theta-\theta_{n} / 2\right)=f_{f_{1} f_{2}}^{n} R_{B}^{(n)}(\theta)
$$


where $f_{i_{1} i_{2}}^{n}$ are vertices satisfying $f_{+-}^{n}(-1)^{n}=f_{-+}^{n}$ and $f_{ \pm \pm}^{n}=0$. Furthermore, the solution of (38) has to satisfy the boundary unitarity and boundary cross-unitarity conditions

$$
\begin{aligned}
& R_{B}^{(n)}(\theta) R_{B}^{(n)}(-\theta)=\mathbb{I}, \\
& R_{B}^{(n)}(i \pi / 2-\theta)=R_{B}^{(n)}(i \pi / 2+\theta) S^{(n, n)}(2 \theta),
\end{aligned}
$$

respectively. Here, $S^{(n, n)}(2 \theta)$ denotes the scattering amplitude for $B_{n}+B_{n} \rightarrow B_{n}+B_{n}$ process [27]. In particular, it possesses a pole in the physical strip located at $\theta=n \pi / 2 \lambda$. It is straightforward to show that

$$
\begin{aligned}
& Y_{0}\left(\theta+\theta_{n} / 2\right) Y_{0}\left(\theta-\theta_{n} / 2\right) \rho(-2 i \theta) / i=-\frac{1}{\sinh (2 \lambda \theta-i \lambda \pi)} R_{0}^{(n)}(u) S^{(n)}(0, u) S^{(n)}(\pi / 2, u), \\
& Y_{1}\left(\theta+\theta_{n} / 2\right) Y_{1}\left(\theta-\theta_{n} / 2\right)=\frac{1}{\sinh (2 \lambda \theta) \sinh (2 \lambda \theta-2 i \lambda \pi)} S^{(n)}(\pi / 2+\pi \lambda / 2, u) S^{(n)}(\pi \lambda / 2, u) .
\end{aligned}
$$

Here we used the notations of $[15,16]$ i.e.

$$
R_{0}^{(n)}(u)=\frac{\left(\frac{1}{2}\right)\left(1+\frac{n}{2 \lambda}\right)}{\left(\frac{3}{2}+\frac{n}{2 \lambda}\right)} \prod_{l=1}^{n-1} \frac{\left(\frac{l}{2 \lambda}\right)\left(1+\frac{l}{2 \lambda}\right)}{\left(\frac{3}{2}+\frac{l}{2 \lambda}\right)^{2}} \quad \text { and } \quad S^{(n)}(x, u)=\prod_{l=0}^{n-1} \frac{\left(\frac{x}{\lambda \pi}-\frac{1}{2}+\frac{n-2 l-1}{2 \lambda}\right)}{\left(\frac{x}{\lambda \pi}+\frac{1}{2}+\frac{n-2 l-1}{2 \lambda}\right)}
$$

where we have the shorthand notation $(x)=\sin (u / 2+x \pi / 2) / \sin (u / 2-x \pi / 2)$. Taking into account the nondiagonal part of the soliton reflection matrix, we finally obtain after some calculations the result

$$
R_{B}^{(n)}(\theta)=(-1)^{n} R_{0}^{(n)}(u) S^{(n)}(0, u) S^{(n)}(\pi / 2, u) S^{(n)}(\pi \lambda / 2+\pi / 2, u) S^{(n)}(\pi \lambda / 2, u)
$$

which, clearly, does not depend on the boundary operators $\hat{\mathcal{E}}_{ \pm}$. As expected, the factor $R_{0}^{(n)}(u)$ contains the poles in the physical strip located at $u_{n}=\pi / 2-n \pi / 2 \lambda$ for $\lambda>1$. They are associated with the breather-breather bound states $B_{2 n}$.

\subsection{Restriction of the boundary state}

Up to now, we only considered the scattering properties without imposing any restriction beyond (7) to the boundary state $|B\rangle$. However, it is necessary to exclude those which would violate certain physical principles, i.e. it must be in accordance with the SG scattering processes. For instance, the term $R_{0}(-i \theta)$ in eq. (35) contains poles in the "physical strip" $0<\theta<i \pi / 2$ located at $\theta_{n}=i n \pi / 2 \lambda ; n=1, \ldots<\lambda$. They are associated with the zero-momentum breathers $B_{n}(0)$ that should appear as bound states of soliton-antisoliton. At these values of the rapidity any off-diagonal entry of (29) which appears in (33) applied to a state $\left|\mathrm{B}_{n}\right\rangle$ will not, in general, give a null result. As breathers are neutral, the state $\left|\mathrm{B}_{n}\right\rangle$ must however be a null vector of $B\left(\theta_{n}\right)$ and $D\left(\theta_{n}\right)$, i.e it must obey the condition

$$
B\left(\theta_{n}\right)\left|\mathrm{B}_{n}\right\rangle=0 \quad \text { and } \quad D\left(\theta_{n}\right)\left|\mathrm{B}_{n}\right\rangle=0
$$

for all $n=1,2, \ldots<\lambda$. In the non-dynamical boundary case [3] this was trivially satisfied due to the form of the off-diagonal entries. Combining these two conditions we obtain

$$
[\cosh \hat{p}, \cosh \hat{q}]\left|\mathrm{B}_{n}\right\rangle=0 \quad \text { and } \quad \cosh \hat{p} \cosh \hat{q}\left|\mathrm{~B}_{n}\right\rangle=(-1)^{n} \cos ^{2}(\pi \lambda)\left|\mathrm{B}_{n}\right\rangle .
$$

To characterize each state $\left|B_{n}\right\rangle$ explicitly, we introduce the one dimensional "space" representation $\{|x\rangle\}$ for the Heisenberg operators defined by

$$
\hat{p}|\mathrm{x}\rangle=-2 i \pi \lambda \partial / \partial \mathrm{x}, \quad \hat{q}|\mathrm{x}\rangle=\mathrm{x}|\mathrm{x}\rangle \quad \text { and } \quad\left\langle x \mid \mathrm{B}_{n}\right\rangle \equiv \Psi_{n}(-i \mathrm{x} / \pi) .
$$

From the first equation in (42), it is straightforward to obtain a difference equation satisfied by $\Psi_{n}(\mathrm{y})$ for $\mathrm{y}=$ $-i \times / \pi$. Obviously, at that stage $n$ does not appear explicitly. It reads

$$
\frac{\Psi_{n}(\mathrm{y}+2 \lambda)}{\Psi_{n}(\mathrm{y}-2 \lambda)}=\frac{\sin (\pi(\mathrm{y}-\lambda))}{\sin (\pi(\mathrm{y}+\lambda))}
$$


Up to an irrelevant overall constant, it can be solved by the integral representation

$$
\Psi_{n}(\mathrm{y})=\exp \int_{0}^{\infty} \frac{d t}{t} \frac{\sinh ^{2}((\mathrm{y}-1 / 2) t)}{\sinh (t) \cosh (2 \lambda t)} \quad \text { for } \quad \mathcal{R} e(\mathrm{y})<1+\lambda
$$

and is defined through analytic continuation otherwise. The second equation will fix the "allowed" values of $y$. It reads

$$
(-1)^{n} 2 \cos ^{2}(\pi \lambda) \Psi_{n}(\mathrm{y})=\cos (\pi \mathrm{y})\left(\Psi_{n}(\mathrm{y}-2 \lambda)+\Psi_{n}(\mathrm{y}+2 \lambda)\right) \quad \text { for } \quad \mathrm{y} \in\left\{\mathrm{y}^{(n)}\right\} .
$$

The condition (46) is divided into two sectors, "odd" $n$ breathers and "even" $n$ breathers. Then, using the definition (45) the boundary state $\left|\mathrm{B}_{n}\right\rangle$ can be decomposed on the finite set of elementary "odd" and "even" states $n \in\{2 p, 2 p+1\}$ with $\left|\mathrm{B}_{n}\right\rangle=\mathrm{N}_{n} \Psi_{n}\left(\mathrm{y}^{(n)}\right)\left|\mathbf{y}^{(n)}\right\rangle \quad\left(\mathrm{N}_{n}\right.$ are some normalization constants) which, due to (33), implies that $K_{S G}^{(\epsilon)}\left(\theta_{n}\right)\left|\mathrm{B}_{n}\right\rangle$ will be automatically block diagonal.

Notice that in the commutative limit $\lambda=0$ in (44) the function $\Psi_{n}(y)$ remains arbitrary and (46) leads to $y=n$. In other words, the boundary state $|B\rangle$ is not restricted anymore in agreement with [3].

\section{Concluding remarks}

As was suggested in [3], boundary integrable quantum field theories including degrees of freedom at the boundary can be constructed. Here, we provide such an example corresponding to a sine-Gordon quantum field theory coupled to a non-linear quantum oscillator at the boundary, which follows the work initiated in [18]. For certain boundary conditions, integrability of the model is preserved at quantum level for an exact relation between the sine-Gordon coupling constant and the boundary quantization length. In particular, this later quantity fixes the overall sign of the boundary perturbation. The corresponding soliton/antisoliton reflection scattering matrix $K_{S G}^{( \pm)}(\theta)$ is constructed explicitly using an extension to the dynamical boundary case of the method based on boundary non-local conserved charges [22, 23]. Bound state reflection matrices are also constructed explicitly. Using some physical constraints, the general form of the boundary state is shown to be restricted.

To study this model further, extensions to the dynamical case of known nonperturbative approaches could be useful. In the non-dynamical case, they have provided an efficient way of studying boundary effects in finite-size system. For instance, TBA analysis [9] or boundary reflection amplitude method [28] from which the effective central charge and boundary ground state energy can be deduced. Comparison of both approaches provides important checks of the exact relation between UV and IR parameters as well as the boundary ground state energy. Here, such methods extended to the dynamical case would obviously provide important information.

Due to the form of our model, one may wonder if it could not be related with certain massive extension of the field theory description of the Kondo model [29]. In [30] two massive versions of the anisotropic spin 1/2 Kondo model have been proposed. It is thus interesting to compare results from [30] (see also [31]) to ours. The first massive Kondo (MK) model is one natural generalization of the integrable massless Kondo model for arbitrary spin studied in [29]. The MK Hamiltonian reads

$$
H_{M K}=\frac{1}{2} \int_{-\infty}^{0} d x\left((\pi(x))^{2}+\left(\partial_{x} \phi(x)\right)^{2}-G \cos (\beta \phi(x))\right)+\lambda_{0}\left(S_{+} e^{i \beta \phi(0) / 2}+S_{-} e^{-i \beta \phi(0) / 2}\right)
$$

where $S_{ \pm}$form a spin $1 / 2$ representation of the $q_{0}$-deformed quantum algebra $s u_{q_{0}}(2)$. The $s u_{q_{0}}(2)$ relation are

$$
\left[S_{z}, S_{ \pm}\right]= \pm 2 S_{ \pm}, \quad\left[S_{+}, S_{-}\right]=\frac{q_{0}^{S_{z}}-q_{0}^{-S_{z}}}{q_{0}-q_{0}^{-1}} \quad \text { and } \quad q_{0}=\exp \left(i \beta^{2} / 8\right) .
$$

This model has been studied in details in [31] at the free fermion point $\beta^{2}=4 \pi$ and in [30] at the reflectionless points for which it was claimed to be integrable. Unlike the massless version, it is not integrable for arbitrary $\beta$. On the other hand, in [30] the modified massive Kondo model (mMK) with Hamiltonian

$$
\begin{aligned}
H^{m M K}=\frac{1}{2} \int_{-\infty}^{0} d x\left((\pi(x))^{2}\right. & \left.+\left(\partial_{x} \phi(x)\right)^{2}-G \cos (\beta \phi(x))\right) \\
& -i \lambda_{0}\left(S_{+} \cos (\beta(\phi(0)-\hat{\phi}) / 2)-S_{-} \cos \left(\beta\left(\phi(0)-\hat{\phi}^{*}\right) / 2\right)\right)
\end{aligned}
$$


was proposed. Here $\hat{\phi}=-\frac{2}{\beta}\left(\frac{\pi}{2}-i \hat{\phi}_{0}\right)$ with $\hat{\phi}_{0}$ a free parameter and the coupling $\lambda_{0}$ is related to the bulk coupling $G$. This model is believed to be integrable for arbitrary $\beta$ and a reflection matrix has been conjectured.

As was mentioned in $[31,30]$, it is important to notice that the analysis supposed that the boundary scattering does not involve boundary operators. Here we didn't assume this condition, which is crucial for our analysis and leads to a new solution (29) of the boundary Yang-Baxter equations. Then, it would be interesting to see in such case if more solutions can be obtained if a "simpler" bulk scattering is considered instead (reflectionless points). Notice that the free fermion point and the reflectionless points are special cases for which our operators (20) $\hat{\mathcal{E}}_{ \pm}$commute, i.e. the $K_{0}$ reflection matrix reduces to Ghoshal-Zamolodchikov and DeVega-Gonzales-Ruiz [3, 24] result (see end of section 3 ).

Other remarks can be made. First, the model mMK is integrable for arbitrary $\beta$ only for a special relation between $G$ and $\lambda_{0}$, and the deformation parameter $q_{0}$ is expressed in terms of the SG coupling constant $\beta$. It is also the case for our model. Secondly, in the MK and mMK models the boundary dependence only occurs in the CDD factors. With the boundary dependence isolated, the massless limit follows by taking the limit $\theta \rightarrow \infty$, which leads to the (massless) Kondo model reflection matrix. However, in our model the boundary dependence occurs through the operators $\cosh (\hat{p}), \cosh (\hat{q})$ in the minimal (non-CDD part) of the reflection matrix (29). Consequently, taking the massless limit of our model requires an analysis which goes beyond the scope of this paper. Finally, for $q$ a root of unity it is easy to construct a finite-dimensional cyclic representation for $\hat{\mathcal{E}}_{ \pm}$. This property might have a certain interest: partition functions of the massless boundary sine-Gordon and the anisotropic Kondo model can be related if the boundary spin is in a cyclic representation [29].

Among other models, it is now quite natural to consider the analytic continuation of the boundary sine-Gordon model studied above. It should provide one of the simplest boundary integrable QFT, the sinh-Gordon model restricted to $x<0$ with quantum degrees of freedom at the boundary. Its spectrum consists of only one particle with mass $m$ and the bulk scattering $S$-matrix for a pair of particles reads [32]

$$
S_{s h G}(\theta)=\frac{(-1)}{\left(\frac{B}{2}\right)\left(1-\frac{B}{2}\right)} \quad \text { where } \quad B=\frac{2 b^{2}}{2+b^{2}} .
$$

In particular, this amplitude is self-dual under the weak-strong coupling duality transformation $b \leftrightarrow 2 / b$. Notice that the bulk $S$-matrices associated with higher simply laced Toda theories enjoy the same property. As was shown in $[12,13,14]$ for non-dynamical integrable boundary conditions, the sinh-Gordon reflection factor obtained from analytic continuation of Ghoshal's result [4] is not self-dual.

Here, taking the analytic continuation $\hat{\beta} \rightarrow i b$ in (40) for $n=1$, the fundamental particle reflection factor takes the simple form

$$
K_{s h G}(\theta)=\frac{\left(-\frac{1}{2}\right)}{\left(\frac{B}{4}\right)\left(\frac{1}{2}-\frac{B}{4}\right)} .
$$

Then, up to an overall sign in front of the boundary perturbation, integrability is ensured for the boundary quantization length (after changing $\hat{\beta} \rightarrow i b$ in $(21)(-)$ ) fixed to

$$
[\hat{p}, \hat{q}]=\alpha_{s h G} \quad \text { with } \quad \alpha_{s h G}=i 4 \pi / B .
$$

Consequently, weak-strong coupling duality of the boundary integrable sinh-Gordon model corresponds to the boundary operator-coupling transformation

$$
\left\{\hat{p}^{*}, \hat{q}^{*}, b^{*}\right\}=\{b \hat{p} / \sqrt{2}, b \hat{q} / \sqrt{2}, 2 / b\} .
$$

Although we didn't discuss them here, it seems to us that our analysis can provide a starting point concerning certain open problems:

- A different choice of trigonometric $R$-matrix gives the Boltzmann weights of the six-vertex model. It is known to be related to the XXZ and the XXX (in its rational limit) spin chains. Applying our result to this case, the value of the boundary quantization length will define the value of the anisotropy which preserves integrability of 
the model. Consequently, we hope our solution $K_{0}(\theta)$ which satisfies the corresponding qBYBEs can be useful in the study of spin chains with dynamical boundary conditions ${ }^{13}$.

- Statistical models with extended line of defects have attracted attention as such inhomogeneities affect the critical properties of the pure statistical systems. In the continuum limit, the scattering theory of their massive excitations is described in terms of the bulk scattering amplitudes as well as those associated with the interaction of particles with the defect line. If integrability is preserved, they reduce to reflection-transmission amplitudes which satisfy the so-called reflection-transmission equations [35]. Similarly to [18] and in the present work, one may be interested in solutions of these equations with quantum boundary degrees of freedom in the defect, taking a reflection matrix of the form (17), (18). We will discuss this problem elsewhere [36].

Acknowledgements: We are very grateful to R. Sasaki for a careful reading of the manuscript and important comments. P.B also thanks P. Dorey, A. Doikou and M. Stanishkov for useful discussions and interest in this work, as well as S. Nicolis and S. Jhingan. P.B thanks hospitality of the "Laboratoire de Mathématiques et Physique Théorique" in Tours where part of this work has been done. K.K thanks hospitality of Yukawa Institute for Theoretical Physics. P.B's work supported by JSPS fellowship.

\section{References}

[1] E.K. Sklyanin, Funct. Anal. Appl. 21 (1987) 164.

[2] E.K. Sklyanin, J. Phys. A 21 (1988) 2375.

[3] S. Ghoshal and A.B. Zamolodchikov, Int. J. Mod. Phys. A 9 (1994) 3841.

[4] S. Ghoshal, Int. J. Mod. Phys. A 9 (1994) 4801.

[5] A. Fring and R. Koberle, Int. J. Mod. Phys. A 10 (1995) 739.

[6] P. Fendley, H. Saleur and N.P. Warner, Nucl. Phys. B 430 (1994) 577.

[7] H. Saleur, S. Skorik and N.P. Warner, Nucl. Phys. B 441 (1995) 421.

[8] S. Skorik and H. Saleur, J. Phys. A 28 (1995) 6605.

[9] A. LeClair, G. Mussardo, H. Saleur and S. Skorik, Nucl. Phys. B 453 (1995) 581.

[10] V.A. Fateev, S. Lukyanov, A.B. Zamolodchikov and Al.B. Zamolodchikov, Phys. Lett. B 406 (1997) 83.

[11] P. Dorey, R. Tateo and G. Watts, Phys. Lett. B 448 (1999) 249.

[12] E. Corrigan, Int. J. Mod. Phys. A 13 (1998) 2709.

[13] E. Corrigan and G.W. Delius, J. Phys. A 32 (1999) 8601.

[14] E. Corrigan and A. Taormina, J. Phys. A 33 (2000) 8739.

[15] P. Mattsson and P. Dorey, J. Phys. A 33 (2000) 9065.

[16] Z. Bajnok, L. Palla, G. Takacs and G.Zs. Toth, Nucl. Phys. B 622 (2002) 548.

[17] Peter Mattsson, Ph.D. thesis, Durham.

[18] P. Baseilhac and G.W. Delius, J. Phys. A 34 (2001) 8259.

[19] V.B. Kuznetsov, J. Phys. A 28 (1995) 4639.

\footnotetext{
${ }^{13}$ We thank A. Doikou for bringing our attention to [33, 34].
} 
[20] V.B. Kuznetsov and A.V. Tsyganov, J. Math. Sci. 80 (1996) 1802.

[21] D. Bernard and A. LeClair, Commun. Math. Phys. 142 (1991) 99.

[22] L. Mezincescu and R.I. Nepomechie, Int. J. Mod. Phys. A 13 (1998) 2747.

[23] G.W. Delius and N. MacKay, "Quantum group symmetry in sine-Gordon and affine Toda field theories on the half-line", submitted to Commun. Math. Phys., hep-th/0112023.

[24] H.J. de Vega and A. González-Ruiz, J. Phys. A 27 (1994) 6129.

[25] T. Hollowood, Nucl. Phys. B 384 (1992) 523.

[26] Al.B. Zamolodchikov, Int. J. Mod. Phys. A 10 (1995) 1125.

[27] A.B. Zamolodchikov and Al.B. Zamolodchikov, Ann. Phys. 120 (1979) 253.

[28] C. Ahn, C. Kim and C. Rim, Nucl. Phys. B 628 (2002) 486.

[29] P. Fendley, F. Lesage and H. Saleur, J. Stat. Phys. 85 (1996) 211;

P. Fendley and H. Saleur, Phys. Rev. Lett. 75 (1995) 4492.

[30] Z.S. Bassi and A. LeClair, Nucl.Phys. B 552 (1999) 643.

[31] A. LeClair, Ann. Phys. 271 (1999) 268.

[32] A.E. Arinshtein, V.A. Fateev and A.B. Zamolodchikov, Phys. Lett. B 87 (1979) 389.

[33] R.I. Nepomechie, Nucl. Phys. B 622 (2002) 615.

[34] A. Doikou and P.P. Martin, "Hecke algebraic approach to the reflection equation for spin chains", hepth/0206076.

[35] G. Delfino, G. Mussardo and P. Simonetti, Phys. Lett. B 328 (1994) 123;

G. Delfino, G. Mussardo and P. Simonetti, Nucl. Phys. B 432 (1994) 518.

[36] P. Baseilhac and K. Koizumi, in preparation. 